\title{
Combination of Fludarabine and Imatinib Induces Apoptosis Synergistically Through Loss of Mitochondrial Membrane Potential and Increases in Caspase-3 Enzyme Activity in Human K562 Chronic Myleloid Leukemia Cells
}

\author{
Baran Yusuf, ${ }^{1}$ Coskun Oztekin, ${ }^{2}$ and Bassoy Esen Yonca ${ }^{1}$ \\ Izmir Institute of Technology, Department of Molecular Biology and Genetics, Urla, Izmir, Turkey ${ }^{1}$ \\ Ankara Numune Education and Research Hospital, Department of Family Medicine, Çankaya, Ankara, Turkey ${ }^{2}$
}

\begin{abstract}
In this study, we aimed to show the synergistic apoptotic effects of imatinib/fludarabine combination in human K562 chronic myleloid leukemia (CML) cells. There was a significant increase in cytotoxicity of combination of imatinib and fludarabine as compared to any agent alone. On the other hand, combination of both agents induced apoptosis significantly as confirmed by increases in caspase-3 enzyme activity and decreases in mitochondrial membrane potential. As a summary, the results of this study strongly suggest that combination of imatinib and fludarabine induced cell death synergistically comparing to only imatinib or fludarabine in human $\mathrm{K} 562 \mathrm{CML}$ cells.
\end{abstract}

\section{INTRODUCTION}

Chronic myeloid leukemia is a hematopoietic stem cell disorder induced by Philadelphia chromosome that results from the reciprocal translocation between chromosome 9 and 22. ABL and BCR genes transferred from the chromosome 9 and 22, respectively, generate the $\mathrm{BCR} / \mathrm{ABL}$ hybrid oncogene. $\mathrm{BCR} / \mathrm{ABL}$ fusion protein has a constitutive tyrosine kinase activity due to the presence of kinase domain of ABL. This fusion protein operates several signaling pathways causing uncontrolled cellular proliferation, reduction of apoptosis, and decrease in the cellular adhesion (1-6).

Keywords: Chronic myeloid leukemia, Imatinib, Fludarabine, $B C R / A B L$, Apoptosis

Correspondence to:

Yusuf Baran

Associate Professor, Izmir Institute of Technology

Department of Molecular Biology and Genetics

Cancer Genetics and Molecular Hematology Laboratory

35430, Urla

Izmir, Turkey

email: yusufbaran@iyte.edu.tr
In recent years, imatinib (imatinib mesylate, STI571, Gleevec) has become a widely used anticancer agent for the treatment of CML patients. Imatinib is able to recognize 21 amino acid in the ATP-binding side of the BCR/ABL and thus can prevent uncontrolled activities of BCR/ABL. It has been responded hematologically and cytogenetically well by the CML patients in the beginning of the treatment but resistance to imatinib observed in the blast crisis phase and in proceeding times has become one of the major problems for the treatment of CML patients $(2,7,8)$. Resistance to imatinib can be divided into two groups as primary and secondary resistance that emerges from the beginning of the treatment and after the treatment, respectively. There are several approaches that have been developed to overcome imatinib resistance. Increment in the imatinib dose, development and application of the other tyrosine kinase inhibitors, or combination of imatinib with other anticancer agents are preferred to overcome imatinib resistance (8).

Fludarabine is a nucleoside purine analogue that inhibits the DNA synthesis and repair (9). Phosphorylated form of fludarabine as 2-F-ara-A is transported into the cell and it is rephosphorylated to 2-F-ara-ATP. This form of fludarabine has the ability to prevent the activity of enzymes involved in DNA synthesis and DNA repair. Inhibition of DNA polymerase alpha, DNA 
primase, ligase, and reductase induces DNA damage and arrest the cell cycle (10-13).

In cancer therapy, it is possible to increase the effect of an agent with another agent and also remission of the resistance by combination therapy. Different agents influencing a variety of oncogenic pathways were used in combination to obtain synergistic cytotoxic effect. For optimizing individual therapy, combination therapy is a significant choice. An exciting research on the mathematical framework to CML shows that a combination of targeted drugs with different specialties can overcome the resistance. By this way, the principles that form the basis of the resistance emergence in cancers treated with targeted drugs with imatinib were shown by mathematical framework (14).

In this study, we tried to increase the sensitivity of human K562 CML cells to imatinib by combination of imatinib with fludarabine and to show the apoptotic mechanisms involved in cell death.

\section{METHODS}

\section{Cell lines and culture conditions}

K562 human CML cells were obtained from the German Collection of Microorganisms and Cell Cultures. These cells were maintained in RPMI 1640 medium containing $10 \%$ fetal bovine serum and $1 \%$ penicillin-streptomycin (Invitrogen, $\mathrm{USA}$ ) at $37^{\circ} \mathrm{C}$ in $5 \% \mathrm{CO}_{2}$.

\section{Determination of cell proliferation by XTT}

IC50 values (dose of the drugs in which $50 \%$ of the cells can survive) of fludarabine and imatinib were determined by XTT as described previously (15). Briefly, $2 \times 10^{4}$ cells were maintained in 96-well plates with $200 \mu \mathrm{L}$ growth medium in the absence or presence of increased doses of fludarabine and imatinib. Then, they were incubated at $37^{\circ} \mathrm{C}$ in $5 \% \mathrm{CO}_{2}$ for $72 \mathrm{hr}$. The cells were treated with $40 \mu \mathrm{l}$ of XTT reagent for more hours and then the plates were read under $490 \mathrm{~nm}$ wavelength by Elisa reader (Thermo Electron Corporation Multiskan Spectrum, Finland). According to the cell survival plots, IC50 values of the drugs were calculated.

\section{Measurement of caspase-3 enzyme activity}

The caspase- 3 colorimetric assay kit (R\&D Systems, USA) was used to determine the caspase- 3 enzyme activity of the cells. At the beginning, cells that had been induced to apoptosis were collected by centrifugation at $1,000 \mathrm{rpm}$ for $10 \mathrm{~min}$. Then, 100 $\mu \mathrm{L}$ of cold lysis buffer (1X) was added to the cells with the aim of resuspending them. After the lysation, cells were kept on ice for $10 \mathrm{~min}$. Then, they were centrifuged at $14,000 \mathrm{rpm}$ for $1 \mathrm{~min}$. Supernatants were taken into new microcentrifuge tubes and $20 \mu \mathrm{L}$ assay buffer (5X), $25 \mu \mathrm{L}$ of sample, $50 \mu \mathrm{L}$ of sterilized water, and $5 \mu \mathrm{L}$ of caspase- 3 colorimetric substrate were added into 96-well plates with the aim of preparing the reaction mixture. The reaction mixture was incubated at $37^{\circ} \mathrm{C}$ in $5 \% \mathrm{CO}_{2}$ for $2 \mathrm{hr}$. Finally, the plate was read under 405 nm wavelength light (Thermo Electron Corporation Multiskan Spectrum, Finland).

\section{Measurement of the loss of mitochondrial membrane potential}

Mitochondrial membrane potential (MMP) of K562 human CML cells was determined using APO LOGIX JC-1 Assay Kit (Cell Technology, USA). First, the cells that had been induced apoptosis were collected by centrifugation at $1,000 \mathrm{rpm}$ for 10 min. Then, supernatants were removed and $500 \mu \mathrm{L}$ of JC-1 dye was added onto the pellets and they were incubated at $37^{\circ} \mathrm{C}$ in $5 \% \mathrm{CO}_{2}$ for $15 \mathrm{~min}$. After the incubation time, they were centrifuged at 1,000 rpm for $5 \mathrm{~min}$. Then, supernatants were removed and the dyed cells were resuspended again with $2 \mathrm{~mL}$ of assay buffer. They were centrifuged for $5 \mathrm{~min}$ at 1,000 rpm. 500 $\mu \mathrm{L}$ assay buffer was added onto the pellets and $150 \mu \mathrm{L}$ from each of them was added into the 96-well plate. The aggregate red form has absorption/emission maxima of 585/590 nm and the green monomeric form has absorption/emission maxima of $510 / 527 \mathrm{~nm}$. The plate was read in these wavelengths by fluorescence Elisa reader (Thermo Varioskan Spectrum, Finland).

\section{Detection of apoptotic cells by by anexinV by flow cytometry}

Apoptotic cell death was evaluated by using AnnexinV/FITC and propidium iodide double staining method based on apoptosis-related cell membrane modifications. First, cells that had been induced to apoptosis were washed with cold phosphate-buffered saline and then resuspended in $1 \mathrm{X}$ binding buffer. One hundred $\mu \mathrm{l}$ from each solution was transferred to microcentrifuge tubes. Five $\mu 1$ Annexin V-FITC and $5 \mu \mathrm{L}$ propidium iodide (PI) were added on the cells. After gentle vortexing, they were incubated for $15 \mathrm{~min}$ at room temperature in the dark. Four hundred $\mu \mathrm{l}$ of $1 \mathrm{X}$ binding buffer was then added into each tube and they were analyzed by flow cytometry (BD Facscanto flowcytometry, Belgium) within $1 \mathrm{hr}$.

\section{RESULTS}

\section{Cytotoxic effect of imatinib and fludarabine is dose dependent on K562 human CML cells}

IC50 values of imatinib and fludarabine were determined by XTT cell proliferation assay. K562 cells were exposed to stepwise increasing doses of drugs for $72 \mathrm{hr}$ and proliferations of the cells were measured. XTT results showed that proliferation of the cells were 12,16 , and $26 \%$ decreased in response to 1,10 , and $100 \mathrm{nM}$ imatinib, respectively (Figure 1). There were 28, $66,74,80$ and $83 \%$ decreases in the proliferation in 1, 5, 10, 100, and $500 \mathrm{nM}$ fludarabine applied K562 cells, respectively (Figure 2). IC50 values of imatinib and fludarabine were calculated as 280 and $3.3 \mathrm{nM}$, respectively. 


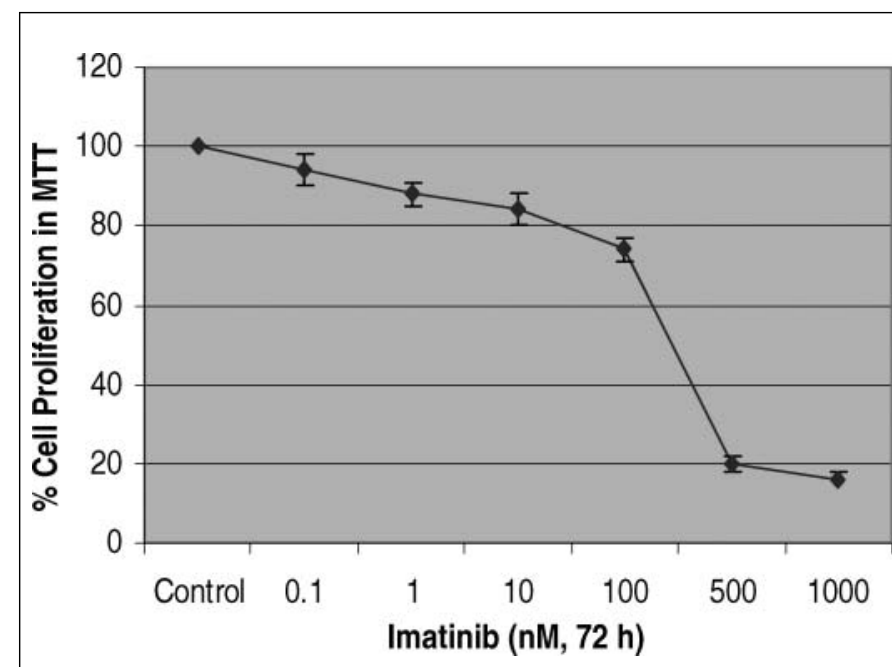

Figure 1. Effects of imatinib on the growth of K562 cells. The IC50 concentration of imatinib was calculated from cell proliferation plots. The XTT assays were performed using triplicate samples in at least two independent experiments. Statistical significance was determined using two-way analysis of variance, and $p<.05$ was considered significant.

\section{Synergistic effect of imatinib and fludarabine combination on K562 human CML cells proliferation}

Proliferation of the cells which were exposed to only $0.1,1$, 10, 100, and $500 \mathrm{nM}$ imatinib was decreased 6, 12, 16, 26, 80, and $84 \%$, respectively, while the application of the same doses of imatinib in combination of $3.3 \mathrm{nM}$ fludarabine decreased the proliferation of K562 cells $80,83,83,85$, and $88 \%$, respectively (Figure 3). These results show that the combination of imatinib and fludarabine has much more cytotoxic effect than any agent alone on K562 human CML cells.

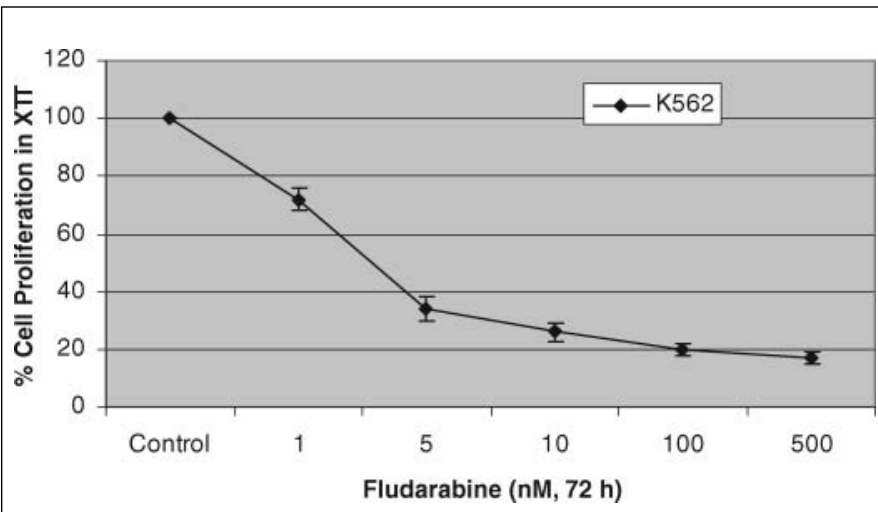

Figure 2. Effects of fludarabine on the growth of K562 cells. The IC50 concentration of fludarabine was determined by XTT assay for each cell line as described. The XTT assays were performed using triplicate samples in at least two independent experiments. Statistical significance was determined using two-way analysis of variance, and $p<.05$ was considered significant.

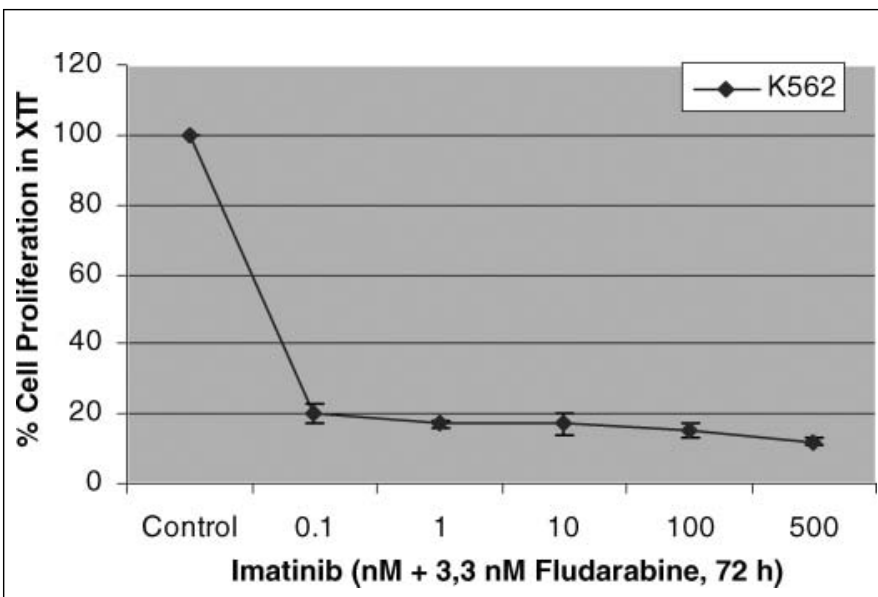

Figure 3. Effects of fludarabine and imatinib combination on the growth of K562 cells. Cytotoxicity was determined by the XTT cell proliferation test in a $72 \mathrm{hr}$ culture. The XTT assays were performed using triplicate samples in at least two independent experiments. Statistical significance was determined using two-way analysis of variance, and $p<.05$ was considered significant.

\section{Synergistic effects of combination of imatinib and fludarabine on the caspase-3 enzyme activity}

As shown in Figure 4, exposures of $3.3 \mathrm{nM}$ fludarabine, 0.1 and $1 \mathrm{nM}$ imatinib alone, increased caspase- 3 enzyme activity 1.36-, 1.16- and 1.26-fold in K562 cells as compared to untreated controls, respectively. On the other hand, there were 1.73- and 1.8-fold increases in caspase- 3 enzyme activity in combination of the same doses of imatinib with $3.3 \mathrm{nM}$ fludarabine comparing to untreated controls, respectively. (Figure 4).

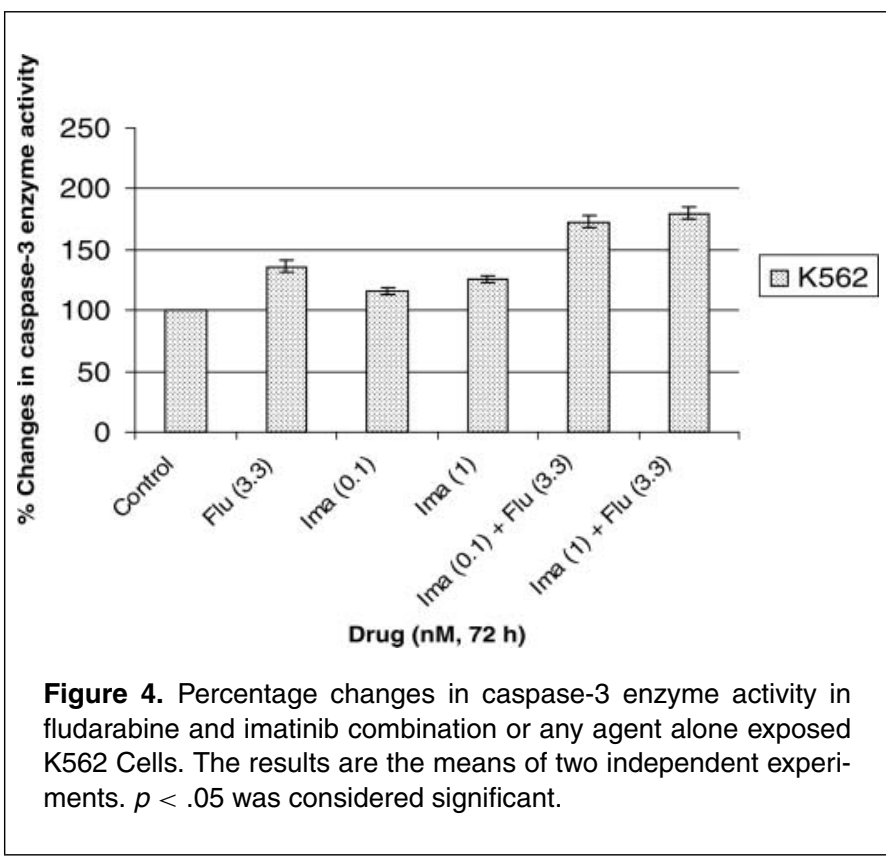




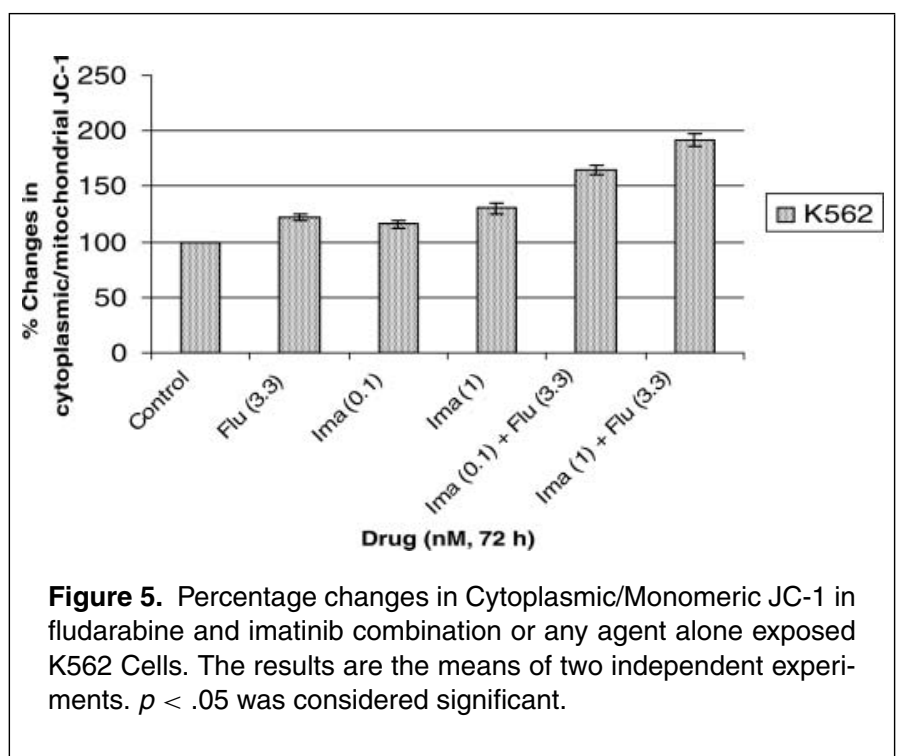

\section{Loss of the mitochondrial memrane potential in response to the combination of imatinib and fludarabine in $\mathrm{K562}$ cells}

Treatment with imatinib/fludarabine combination caused a significant loss of MMP, as measured by increased accumulation of cytoplasmic/monomeric form of JC-1, in K562 cells as compared with any agent alone. There were 1.16- and 1.30fold increases in cytoplasmic/monomeric JC-1 in 0.1 and 1 nM imatinib-applied K562 cells, respectively, while $3.3 \mathrm{nM}$ fludarabine increased cytoplasmic/mitochondrial 1.22 fold by itself (Figure 5). On the other hand, the same concentrations of imatinib with $3.3 \mathrm{nM}$ fludarabine increased cytoplasmic/mitochondrial JC-1 1.65- and 1.92-fold as compared to untreated control group, respectively. (Figure 5).

\section{Imatinib and fludarabine combination induced cell death in CML cells more than any agent alone}

Apoptosis and cell viability in K562 cells were measured by AnnexinV-FITC staining by flow cytometry. There were 30, 22 , and $23 \%$ increases in cell death in response to $3.3 \mathrm{nM}$ fludarabine, 0.1 and $1 \mathrm{nM}$ imatinib in K562 cells, respectively. Exposure of K562 cells to the combination of $3.3 \mathrm{nM}$ fludarabine and 0.1 or $1 \mathrm{nM}$ imatinib increased the percentage of cell death to 36 , and $42 \%$, respectively, as compared to untreated controls [Figures 6(A) and 6(B)].

\section{DISCUSSION}

Inhibition of $\mathrm{BCR} / \mathrm{ABL}$ constitutive tyrosine kinase activity is an important breakthrough in the treatment of CML patients. While imatinib is a well-tolerated agent, it shows very high rates of hematological and cytogenetical responses. However, the emergence of resistance to imatinib has been recognized as a major problem for the treatment of CML patients. Combination of the imatinib with other nonoverlapping mechanism of action combination therapies is a good alternative strategy to manage the resistance problem (16).

Fludarabine, a purine nucleoside analogue, inhibits replication of DNA through interacting not only with DNA polymerase but also with ribonucleotide reductase (17). Fludarabine has shown significant cytotoxicity as it is combined with cyclophosphamide, mitoxantrone, dexamethasone, and rituximab for the treatment of non-Hodgkins lymphomas. On the other hand, fludarabine has also been used for the treatment of acute myeloid leukemia in combination with cytarabine and granulocyte colony-stimulating factor $(18,19)$. In the same way, our results have shown that both imatinib and fludarabine inhibited proliferation of K562 CML cells in a dose-dependent manner. Both of the agents have very high cytotoxic effects even in nanomolar concentrations. IC50 value of imatinib and fludarabine were calculated to be $280 \mathrm{nM}$ and $3.3 \mathrm{nM}$ in K562 cells, respectively. There are some recent researches, which indicate accelerated synergistic apoptotic effects of imatinib and/or fludarabine with a combination of other anticancer agents in hematological malignancies. Effectiveness of imatinib was increased synergistically with lonafarmib for the treatment of patients with CML. The importance of this study comes from the failure of patients, when the imatinib was used as a single agent. Usage of lonafarmib for patients who shows resistant to imatinib is well tolerated by the patients (20). Combination of imatinib with mitoxantrone/etoposide or cytarabine also have been used by Fruehauf and his colleagues and their results revealed that combination of these agents make CML patients more sensitive to imatinib (21). On the other hand, combination of fludarabine with mylotarg, cytarabine (Ara-C), or cyclosporine has significant cytotoxicity on acute myelogenous leukemia and acute lymphoblastic leukemia patients (22). Adaphostin, oxaliplatin, or cyclophosphamide in combination with fludarabine was also shown to induce apoptosis significantly in chronic lymphocytic leukemia (CLL) cells $(23,24)$. In this study, we combined $3.3 \mathrm{nM}$ fludarabine with increasing concentrations of imatinib and observed synergistic antiproliferative effects on K562 cells. While imatinib could not show cytotoxic effects on $\mathrm{K} 562$ cells in $0.1,1,10$, and $100 \mathrm{nM}$ concentrations, combination of these doses of imatinib, with $3.3 \mathrm{nM}$ fludarabine resulted in more that $80 \%$ reduction in proliferation of $\mathrm{K} 562$ cells. In parallel with our experimental results, Korycka and colleagues have shown that there were synergistic cytotoxic effects of imatinib in combination with cladribine (2-CdA), a purine analogue, on the normal and chronic myelogenous leukemia progenitor cells. It was clearly shown by this study that imatinib together with 2-CdA at different concentration combinations significantly blocked the colony growth of CML granulocytemacrophage progenitor cells (CFU-GM), as compared either to the control or to any agent used alone (25). We conducted three more molecular approaches in order to confirm the synergistic effects of imatinib and fludarabine in addition to XTT cell proliferation data. The results of changes in caspase- 3 enzyme activity, loss of mitochondrial membrane potential, and changes 

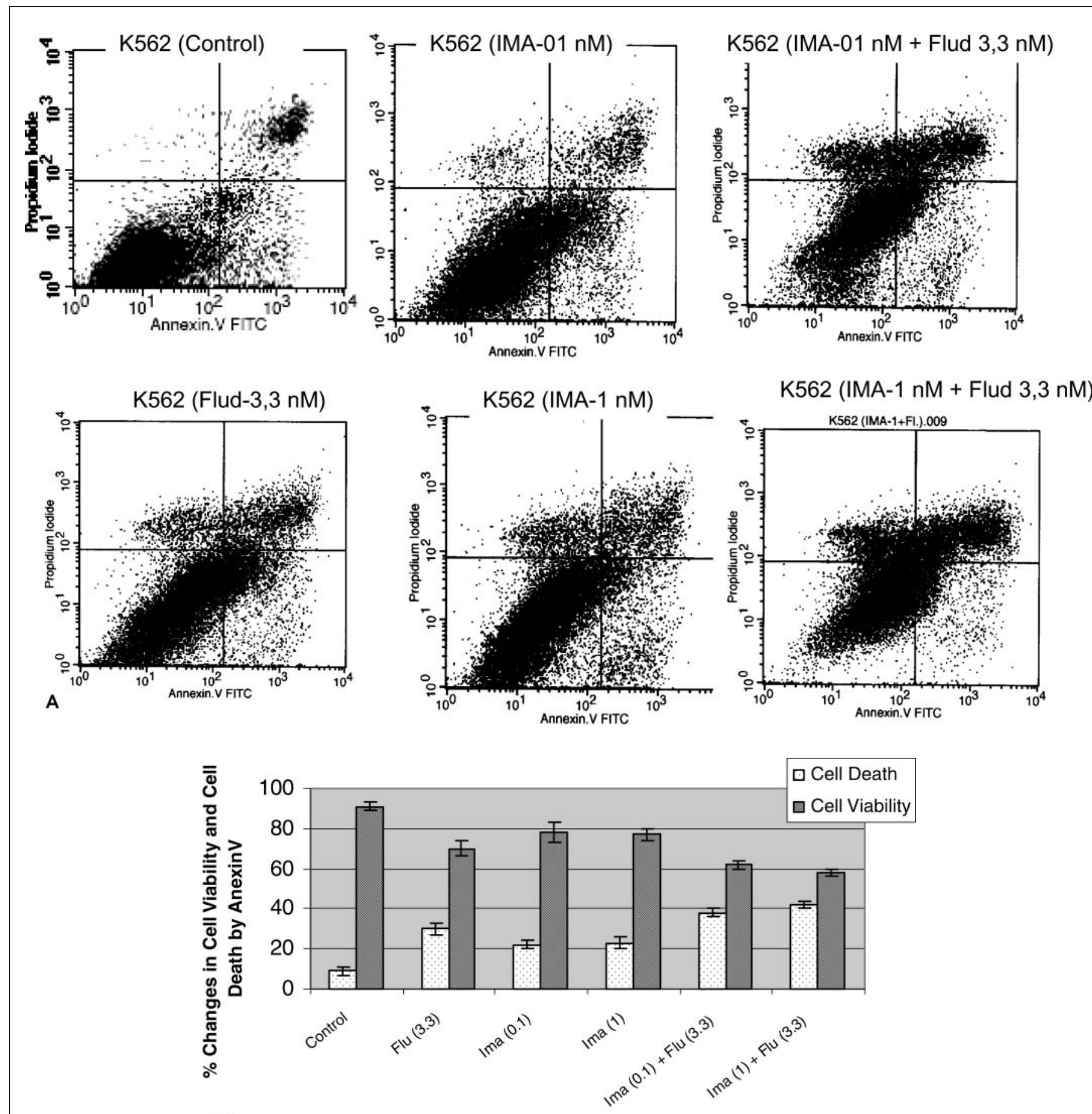

B

Drugs (nM, $72 \mathrm{~h})$

Figure 6. Percentage changes in cell death by anexinV-FITC in imatinib and fludarabine combination or any agent alone exposed K562 cells. The results are the means of two independent experiments. The error bars represent the standard deviations, and when not seen, they are smaller than the thickness of the lines on the graphs. $p<.05$ was considered significant.

in cell death and viability assays showed in agreement that combinations of very low subtoxic concentrations of imatinib $(0.1$ and $1 \mathrm{nM}$ ) in combination with $3.3 \mathrm{nM}$ fludarabine resulted in synergistic apoptotic effects on K562 cells as compared to any agent alone. The results of caspase- 3 enzyme activity and mitochondrial membrane potential experiments also revealed that combination of these agents induce apoptosis through MMP and caspase-3-dependent pathway. 
In this study, we examined possible synergistic apoptotic effects of imatinib and fludarabine and the mechanisms responsible for induction of apoptosis in human K562 CML cells. While fludarabine inhibits replication of DNA, imatinib inhibits all the signaling pathways regulated by oncogenic BCR/ABL. Our data suggested that fludarabine elevated the chemosensitivity of $\mathrm{BCR} / \mathrm{ABL}$ positive $\mathrm{K} 562$ cells to imatinib. This is most probably because inhibition of replication of DNA besides inhibiton of strong oncogene, BCR/ABL, created strong synergism. This in vitro data by being supported with clinical data may open the way of more effective treatment of CML.

\section{ACKNOWLEDGMENT}

We thank to Dr. Halil Ates for his help during the study and Izmir Institute of Technology, Biotechnology and Bioengineering Research Facility staff for their help and technical support.

\section{DECLARATION OF INTEREST}

The authors report no conflicts of interest. The authors alone are responsible for the content and writing of the paper.

\section{REFERENCES}

1. Ramirez, P.; DiPersio, J.F. Therapy options in imatinib failures. Oncologist 2008, 13, 424-434.

2. Baran, Y.; Salas, A.; Senkal, C.E.; Gunduz, U.; Bielawski, J.; Obeid, L.M.; Ogretmen, B. Alterations of ceramide/sphingosine 1-phosphate rheostat involved in the regulation of resistance to imatinib-induced apoptosis in K562 human chronic myeloid leukemia cells. J Biol Chem 2007, 282, 10922-10934.

3. Cang, S.; Liu, D. P-loop mutations and novel therapeutic approaches for imatinib failures in chronic myeloid leukemia. J Hematol Oncol 2008, 1, 15.

4. Steinberg, M. Dasatinib: a tyrosine kinase inhibitor for the treatment of chronic myelogenous leukemia and Philadelphia chromosomepositive acute lymphoblastic leukemia. Clin Ther 2007, 29, 2289-2308.

5. Sallmyr, A.; Fan, J.; Rassool, F.V. Genomic instability in myeloid malignancies: increased reactive oxygen species (ROS), DNA double strand breaks (DSBs) and error-prone repair. Cancer Lett 2008, 270, 1-9.

6. Koca, E.; Haznedaroglu, I.C. Imatinib mesylate and the management of chronic myeloid leukemia (CML). Turkish $\mathrm{J}$ Hematology 2005, 22(4), 161-172.

7. Jakubowska, J.; Wasowska-Lukawska, M.; Czyz, M. STI571 and morpholine derivative of doxorubicin collaborate in inhibition of K562 cell proliferation by inducing differentiation and mitochondrial pathway of apoptosis. Eur J Pharmacol 2008, 596, 41-49.

8. Henkes, M.; Van Der Kuip, H.; Aulitzky, W.E. Therapeutic options for chronic myeloid leukemia: focus on imatinib (Glivec, Gleevectrade mark). Ther Clin Risk Manag 2008, 4, 163-187.

9. Ding, X.; Herzlich, A.A.; Bishop, R.; Tuo, J.; Chan, C.C. Ocular toxicity of fludarabine: a purine analog. Expert Rev Ophthalmol 2008, 3, 97-109.

10. Nitsche, M.; Christiansen, H.; Hermann, R.M.; Lucke, E.M.; Peters, K.; Rave-Frank, M.; Schmidberger, H.; Pradier, O. The combined effect of fludarabine monophosphate and radiation as well as gemcitabine and radiation on squamous carcinoma tumor cell lines in vitro. Int J Radiat Biol 2008, 84, 643-657.
11. Clavio, M.; Venturino, C.; Pierri, I.; Garrone, A.; Miglino, M.; Canepa, L.; Balleari, E.; Balocco, M.; Michelis, G.L.; Ballerini, F.; Gobbi, M. Combination of liposomal daunorubicin (DaunoXome), fludarabine, and cytarabine (FLAD) in patients with poor-risk acute leukemia. Ann Hematol 2004, 83, 696-703.

12. Harned, T.M.; Gaynon, P.S. Treating refractory leukemias in childhood, role of clofarabine. Ther Clin Risk Manag 2008, 4, 327-336.

13. Rosato, R.R.; Almenara, J.A.; Maggio, S.C.; Coe, S.; Atadja, P.; Dent, P.; Grant, S. Role of histone deacetylase inhibitor-induced reactive oxygen species and DNA damage in LAQ-824/fludarabine antileukemic interactions. Mol. Cancer Ther 2008, 7, 3285-3297.

14. Komarova, N.L.; Wodarz, D. Drug resistance in cancer: principles of emergence and prevention. Proc Natl Acad Sci 2005, 102, 9714-9719.

15. Baran, Y.; Ural, A.U.; Gunduz, U. Mechanisms of cellular resistance to imatinib in human chronic myeloid leukemia cells. Hematology 2007, 12, 497-503.

16. Soverini, S.; lacobucci, I.; Baccarani, M.; Martinelli, G. Targeted therapy and the T315I mutation in Philadelphia-positive leukemias. Haematologica 2007, 92, 437-439.

17. Robak, T. Recent progress in the management of chronic lymphocytic leukemia. Cancer Treat Rev 2007, 33, 710-728.

18. Cortes, J.; Jabbour, E.; Daley, G.Q.; O'Brien, S.; Verstovsek, S.; Ferrajoli, A.; Koller, C.; Zhu, Y.; Statkevich, P.; Kantarjian, H. Phase 1 study of lonafarnib ( $\mathrm{SCH} 66336)$ and imatinib mesylate in patients with chronic myeloid leukemia who have failed prior single-agent therapy with imatinib. Cancer 2007, 110, 1295-1302.

19. Rai, K.R.; Peterson, B.L.; Appelbaum, F.R.; Kolitz, J.; Elias, L.; Shepherd, L.; Hines, J.; Threatte, G.A.; Larson, R.A.; Cheson, B.D.; Schiffer, C.A. Fludarabine compared with chlorambucil as primary therapy for chronic lymphocytic leukemia. $\mathrm{N}$ Engl J Med 2000, 343, 1750-1757.

20. Tournilhac, O.; Cazin, B.; Leprètre, S.; Diviné, M.; Maloum, K.; Grosbois, B.; Feugier, P.; Maloisel, F.; Villard, F.; Villemagne, B.; Bastit, D.; Belhadj, K.; Azar, N.; Michallet, M.; Manhès, G.; Travade, P. Impact of frontline fludarabine and cyclophosphamide combined treatment on peripheral blood stem cell mobilization in B-cell chronic lymphocytic leukemia. Blood 2004, 103, 363365.

21. Fruehauf, S.; Topaly, J.; Buss, E.C.; Fischer, T.; Ottmann, O.G.; Emmerich, B.; Müller, M.C.; Schuld, P.; Balleisen, L.; Hehlmann, R.; Ho, A.D.; Hochhaus, A. Imatinib combined with mitoxantrone/etoposide and cytarabine is an effective induction therapy for patients with chronic myeloid leukemia in myeloid blast crisis. Cancer 2007, 109, 1543-1549.

22. Tsimberidou, A.M.; Estey, E.; Cortes, J.E.; Garcia-Manero, G.; Faderl, S.; Verstovsek, S.; Thomas, D.A.; Ferrajoli, A.; Keating, M.J.; O'Brien, S.; Kantarjian, H.M.; Giles, F.J. Mylotarg, fludarabine, cytarabine (ara-C), and cyclosporine (MFAC) regimen as post-remission therapy in acute myelogenous leukemia. Cancer Chemother Pharmacol 2003, 52, 449-452.

23. Shanafelt, T.D.; Lee, Y.K.; Bone, N.D.; Strege, A.K.; Narayanan, V.L.; Sausville, E.A.; Geyer, S.M.; Kaufmann, S.H.; Kay, N.E. Adaphostin-induced apoptosis in CLL B cells is associated with induction of oxidative stress and exhibits synergy with fludarabine. Blood 2005, 105, 2099-2106.

24. Moufarij, M.A.; Sampath, D.; Keating, M.J.; Plunkett, W. Fludarabine increases oxaliplatin cytotoxicity in normal and chronic lymphocytic leukemia lymphocytes by suppressing interstrand DNA crosslink removal. Blood 2006, 108, 4187-4193

25. Korycka, A.; Robak, T. The influence of imatinib mesylate (STI571) used alone or in combination with purine nucleoside analogues on the normal and chronic myelogenous leukaemia progenitor cells in vitro. Leuk Lymphoma 2003, 44, 15491555. 